\title{
Three epochs of EVN observations towards IRAS $23365+3604$
}

\author{
Cristina Romero-Cañizales* ${ }^{* \dagger}$ \\ Instituto de Astrofísica de Andalucía - CSIC, 18008 Granada, Spain \\ E-mail: cromeroliaa.es
}

Miguel Ángel Pérez-Torres

Instituto de Astrofísica de Andalucía - CSIC, 18008 Granada, Spain

E-mail: torres@iaa.es

\section{Antxon Alberdi}

Instituto de Astrofísica de Andalucía - CSIC, 18008 Granada, Spain

E-mail: antxon@iaa.es

\begin{abstract}
The European VLBI Network (EVN) provides us with the necessary sensitivity and angular resolution to study the nuclear and circumnuclear regions in Luminous $\left(\mathrm{L}_{\mathrm{FIR}}>10^{11} \mathrm{~L}_{\odot}\right)$ and Ultraluminous ( $\mathrm{L}_{\mathrm{FIR}}>10^{12} \mathrm{~L}_{\odot}$ ) Infrared Galaxies. The high Star Formation Rates (SFR) inferred for these galaxies implies both the presence of a high number of massive stars and a dense surrounding medium. Therefore, bright radio $\mathrm{SNe}$ are expected to occur. With the aim of estimating the SFR in ULIRGs by means of Core Collapse supernova (CCSN) detections, we started an observing campaign with the EVN on a small sample of the brightest and farthest ULIRGs in the local Universe. We present here our results from three epochs of quasi-simultaneous observations with the EVN at 6 and $18 \mathrm{~cm}$ towards one of the objects in our sample: IRAS 23365+3604.
\end{abstract}

10th European VLBI Network Symposium and EVN Users Meeting: VLBI and the new generation of radio arrays

September 20-24, 2010

Manchester, $U K$

\footnotetext{
* Speaker.

${ }^{\dagger}$ We acknowledge support from grant AYA2009-13036-C02-01, sponsored by the Spanish MICINN. M.A.P.T acknowledges support from the Autonomic Government of Andalusia under grants P08-TIC-4075 and TIC-126. This research has also benefited from research funding from the European Community Framework Programme 7, Advanced Radio Astronomy in Europe, grant agreement no.: 227290. Thanks to JIVE and especially to Zsolt Paragi and Bob Campbell for their invaluable help in this project. The European VLBI Network is a joint facility of European, Chinese, South African and other radio astronomy institutes funded by their national research councils.
} 


\section{IRAS $23365+3604$}

IRAS $23365+3604\left(\alpha=23^{h} 39^{m} 01.7, \delta=36^{\circ} 21^{\prime} 14^{\prime \prime}\right)$, hereafter IRAS 2336 , is an advanced merger at a distance of $252 \mathrm{Mpc}(1$ mas $\approx 0.8 \mathrm{pc})$ with a very high luminosity $\left(\log \left(\mathrm{L}_{\mathrm{FIR}} / \mathrm{L}_{\odot}\right)=\right.$ 12.13) which corresponds to a CCSN rate of $\approx 5 \mathrm{SN} /$ year [1].

\section{Observations and their analysis}

We present multi-epoch, -frequency EVN observations of the ULIRG IRAS 2336 (see Figure 1). Putative young radio $\mathrm{SNe}$ with typical peak luminosities of $\mathrm{L} \sim 10^{27-28} \mathrm{erg} \mathrm{s}^{-1} \mathrm{~Hz}^{-1}$ should have a flux of 0.01 to $0.1 \mathrm{mJy}$ at the distance of IRAS 2336. The imaging process and therefore the analysis of the observations has proved to be a very challenging task due to:

- The presence of strong extended emission $\left(\sim 10 \mathrm{mJy}, \Theta=00^{\prime \prime} 5\right)$ which hinders the detection of compact sources $(\mathrm{SNe})$.

- The lack of short baselines, which make the imaging algorithm to fail (compare the images we present here with the images we presented in the previous EVN Symposium [2]).

The extended emission at the distance of IRAS 2336 cannot be resolved out with the available angular resolution, and the only way to properly map it is by having a good combination of short baselines (which give enough information for closure phase) and/or using a combination of Gaussian model fitting and delta components. This can be done within the Caltech imaging programme DIFMAP [3], which we have used for the imaging process. We then exported the clean images back into AIPS to analyse them and to produce the final maps that we present here.

\section{Results}

Our images reveal the presence of a nuclear starburst with an approximate extension of $200 \mathrm{pc}$. We note that at $18 \mathrm{~cm}$, the size of the nuclear region is larger than at $6 \mathrm{~cm}$, for all the epochs. This can be explained by the longer lifetime of electrons at lower frequencies [4]. The high luminosities of the nuclear region (see Table 1) can only be explained by the presence of non-thermal emitters most likely SNe- within extended emission. In fact, the flattening (from the first to the third epoch) of the spectral index from the nuclear zone (see Figure 2) can be explained by the variation in flux of sources therein and/or appearance of new sources $(\mathrm{SN})$ which would be seen first at higher frequencies and later at lower frequencies [5]. We have also detected some sources at $18 \mathrm{~cm}$ with emission $>5 \sigma\left(\mathrm{L} \sim 10^{27-28} \mathrm{erg} \mathrm{s}^{-1} \mathrm{~Hz}^{-1}\right)$ with no counterpart at $6 \mathrm{~cm}$. These are candidates of $\mathrm{CCSNe}$ exploding in the circumnuclear region (see sources labelled as probable SNe in Figure 1).

\section{Conclusions}

The EVN has aided to obtain the deepest and highest resolution radio images ever of one of the most distant ULIRGs in the local Universe. High resolution is important, but the information provided by short baselines is also necessary to properly map the morphology of the diffuse emission in this kind of sources. 


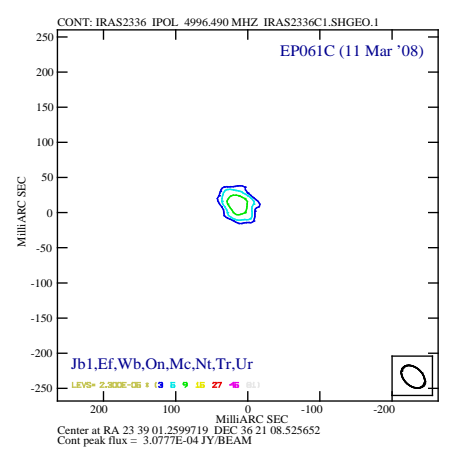

(a)

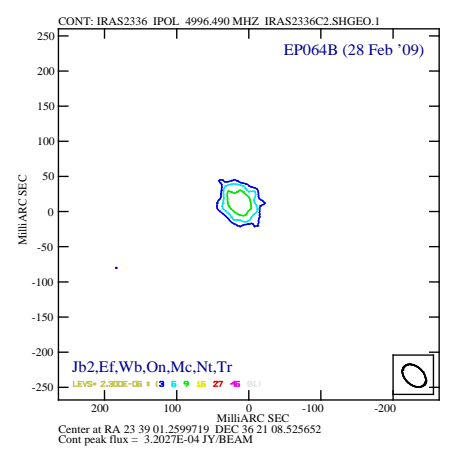

(d)

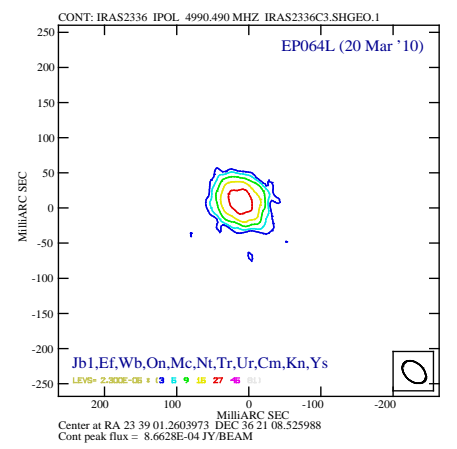

(g)

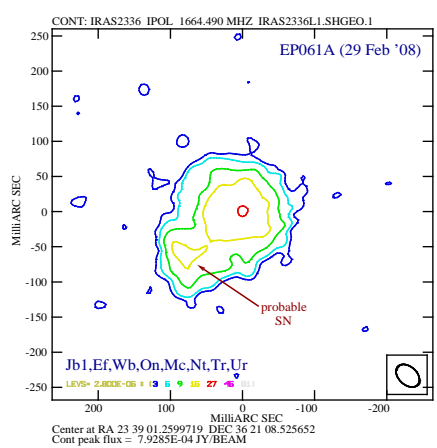

(b)

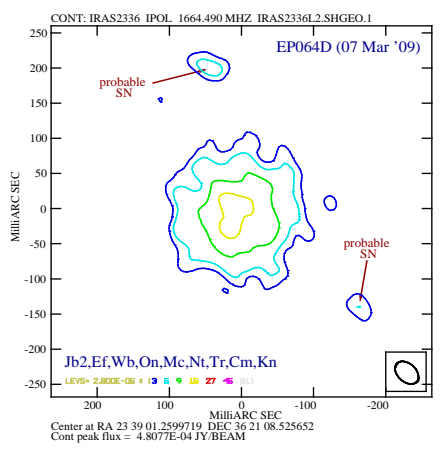

(e)

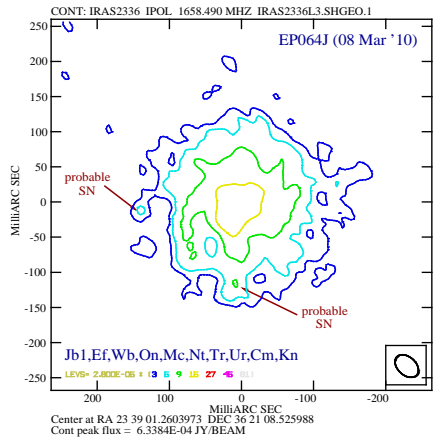

(h)

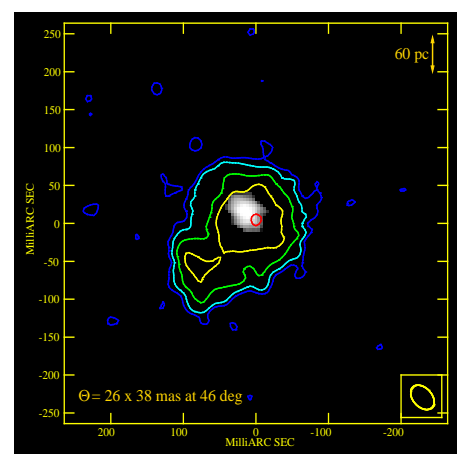

(c)

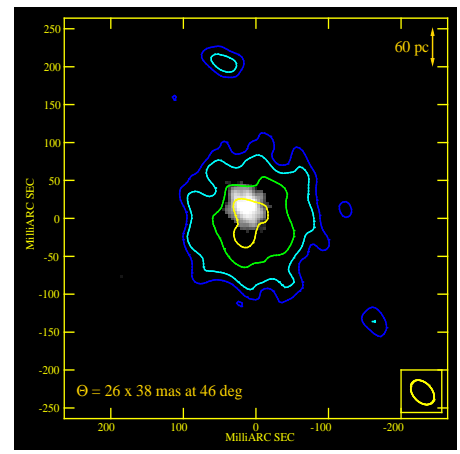

(f)

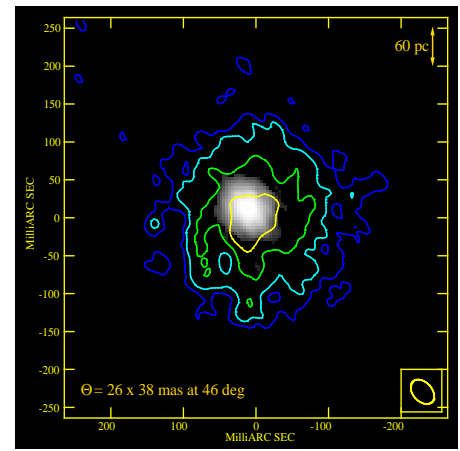

(i)

Figure 1: IRAS 2336 at $6 \mathrm{~cm}$ (left), $18 \mathrm{~cm}$ (middle) and $18 \mathrm{~cm}$ contours overlaid on grey scale $6 \mathrm{~cm}$ images (right), in three different epochs (top to bottom): March 2008, February 2009 and March 2010. All the images have been degraded to the epoch with lowest resolution $(18 \mathrm{~cm}$, second epoch), and thus convolved with a beam size $\sim 30 \times 45$ mas. The lowest contours are $\sim 70$ and $\sim 85 \mu \mathrm{Jy}$ at 6 and $18 \mathrm{~cm}$, respectively. Sources labelled as probable SN in Figures (b), (e) and (h), are $>5 \sigma$ detections and have inferred luminosities $\sim 10^{27-28} \mathrm{erg} \mathrm{s}^{-1} \mathrm{~Hz}^{-1}$. 


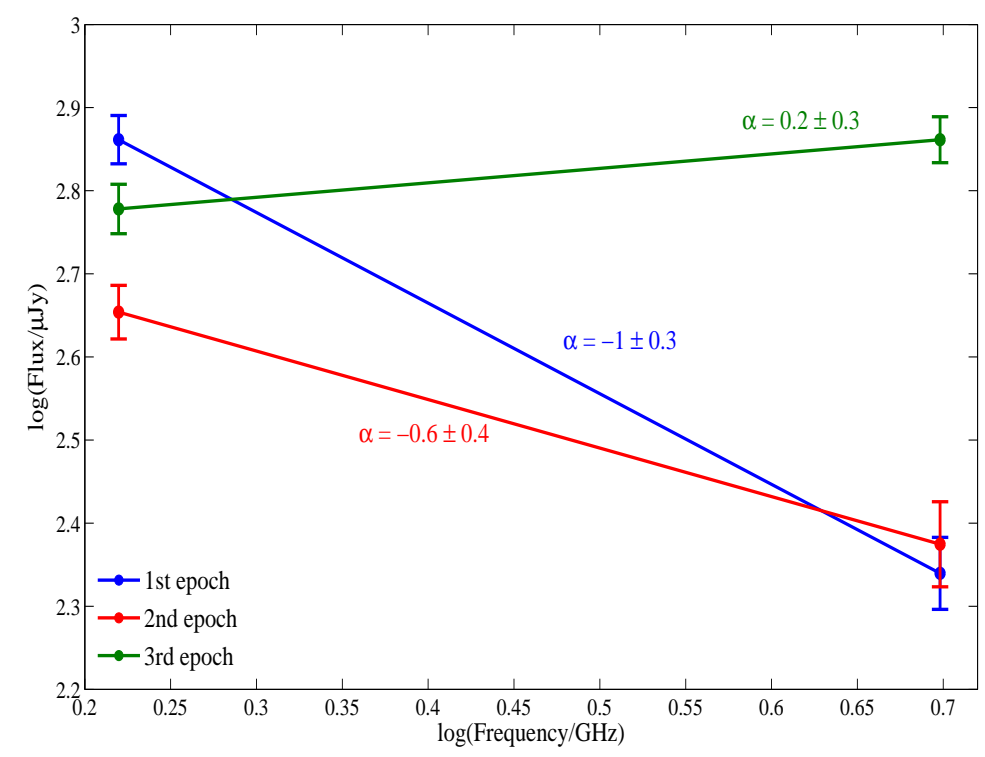

Figure 2: Spectral index $\left(S \sim v^{\alpha}\right)$ evolution of the peak component in the nuclear zone, which we expect to be composed by non-thermal sources (e.g. SNe).

\begin{tabular}{|c|c|c|c|c|}
\hline \multirow{2}{*}{ Epoch } & \multicolumn{2}{|c|}{$\mathrm{L}_{6 \mathrm{~cm}} \times 10^{28} \mathrm{erg} \mathrm{s}^{-1} \mathrm{~Hz}^{-1}$} & \multicolumn{2}{c|}{$\mathrm{L}_{18 \mathrm{~cm}} \times 10^{28} \mathrm{erg} \mathrm{s}^{-1} \mathrm{~Hz}^{-1}$} \\
\cline { 2 - 5 } & peak & total & peak & total \\
\hline 1st & $1.7 \pm 0.2$ & $1.7 \pm 0.2$ & $5.5 \pm 0.4$ & $17.6 \pm 0.4$ \\
2nd & $1.8 \pm 0.2$ & $2.4 \pm 0.2$ & $3.4 \pm 0.3$ & $18.9 \pm 0.3$ \\
3rd & $5.5 \pm 0.4$ & $7.2 \pm 0.4$ & $4.6 \pm 0.3$ & $15.8 \pm 0.3$ \\
\hline
\end{tabular}

Table 1: Peak and total luminosities of the nuclear zone at $6 \mathrm{~cm}$ and $18 \mathrm{~cm}$ in the three different epochs.

\section{References}

[1] Condon, J. J. 1992, ARA\&A, 30, 575

[2] Romero-Cañizales, C., Pérez-Torres, M., \& Alberdi, A. 2008, EVN observations of the Ultra Luminous Infrared Galaxies IRAS 23365+3604 and IRAS 07251-0248 in proceedings of The 9th European VLBI Network Symposium, PoS (IX EVN Symposium) 089

[3] Shepherd, M. C., Pearson, T. J., \& Taylor, G. B. 1994, BAAS, 26, 987

[4] Pacholczyk A. G. 1970, Radio Astrophysics (Freeman \& Co., San Francisco)

[5] Weiler, K. W., Panagia, N., Montes, M. J., \& Sramek, R. A. 2002, ARA\&A, 40, 387 